\title{
Exophytic glioblastoma multiforme originating from the medulla oblongata
}

\author{
Yoshifumi Horita, Masahiko Wanibuchi*, Yukinori Akiyama, Kengo Suzuki, Yoshinori Omori, Satoshi Iihoshi, Satoko Ochi, Takeshi Mikami, \\ and Nobuhiro Mikuni
}

Department of Neurosurgery, Sapporo Medical University, Japan

\begin{abstract}
Glioblastoma multiforme (GBM) is most frequently located in the supratentorial region of the brain. In this paper, we report the case of a 25 -year-old man who presented with a heterogeneous tumor with exophytic features and located in the caudal fourth ventricle. The tumor was subtotally resected and the patient underwent radiotherapy at a dosage of $50 \mathrm{~Gy}$ and concurrent chemotherapy with temozolomide. Histopathological examination revealed the typical features of conventional GBM. The patient independently performed the activities of daily living for 11 months. However, after identifying a recurrence of the tumor in the dorsal medulla oblongata, the patient suddenly died at home 12 months after diagnosis, likely due to respiratory arrest. To the best of our knowledge, it is extremely rare for GBM to occur in the medulla oblongata and only eight cases have been described in detail. Patients with intrinsic GBM of the medulla oblongata died within three months. In contrast, patients with exophytic GBMs of the medulla oblongata survived for more than one year. Patients with GBMs that occurred in the medulla oblongata and exhibited exophytic growth patterns had a better prognosis than patients with intrinsic GBMs because the tumors could be more radically and safely resected.
\end{abstract}

\section{Introduction}

Glioblastoma multiforme (GBM) is most frequently found in the supratentorial region of the brain, but in less than $4 \%$ of cases it can occur in the posterior fossa [1]. In the infratentorial region, GBM usually arises in the pons [2]. GBM occurring in the medulla oblongata is extremely rare. GBM in the medulla oblongata has only been described in detail in eight cases. In this paper, we report on the surgical treatment and outcome of a patient with a GBM arising from the medulla oblongata that exhibited an exophytic growth pattern to the fourth ventricle.

\section{Case report}

A 25-year-old man presented with a progressive headache. Neurological examination of the patient showed mild dysarthria and dysphagia. The patient also had sporadic singultus. The patient had a history of bipolar disorder that was diagnosed when he was 20 years old. Preoperative magnetic resonance imaging (MRI) showed a heterogeneous and ring-like enhanced mass after administration of gadolinium-diethylenetriamine pentaacetic acid (Gd-DTPA). The mass appeared hypointense in a T1-weighted image and hyperintense in a T2-weighted image of the lower half of the fourth ventricle and the foramen of Magendie (Figures 1A, 1 B). The tumor had an exophytic growth pattern and had dimensions of $28 \times 35 \times 30 \mathrm{~mm}$ (Figures $1 \mathrm{C}$, 1D). Cerebral angiography demonstrated that the bilateral posterior inferior cerebellar arteries provided blood to the tumor.

The tumor was approached via a median suboccipital craniotomy with additional removal of the C-1 arch. After opening of the dura mater, a yellow/red-colored tumor containing many vascular structures was observed. Under intraoperative electrophysiological monitoring, a microsurgical excision was carefully performed. During this procedure, the exophytic character of the tumor became apparent. Intraoperative histopathology suggested that the tumor was a malignant glioma. Since the tumor was tightly attached to the medulla oblongata and to the bilateral posterior inferior cerebellar arteries, a subtotal excision was achieved.

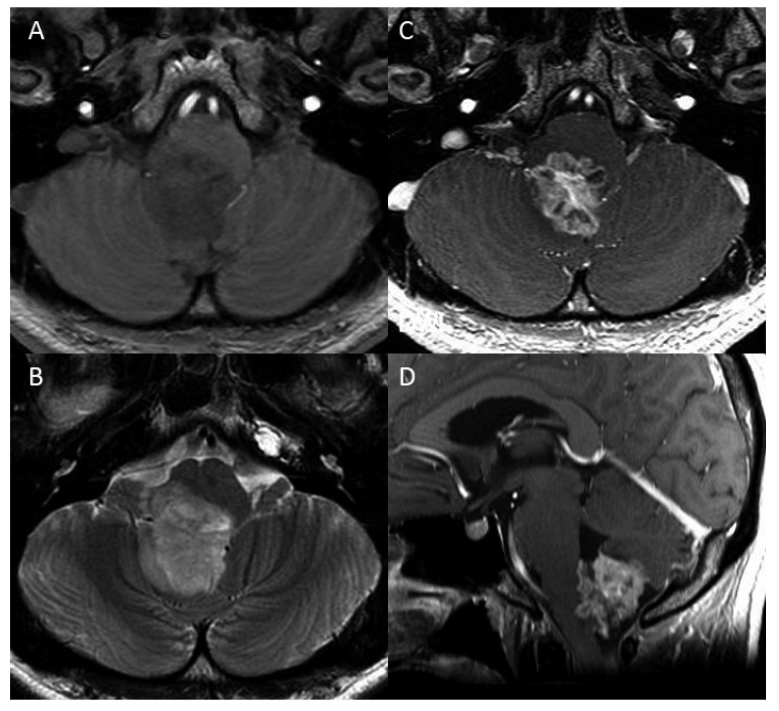

Figure 1. Preoperative magnetic resonance (MR) images: A. The tumor appears hypointense in a T1-weighted image. B. A T2-weighted image shows hyperintense tumor. C, D. Contrast-enhanced MR images show exophytic features that is located in the fourth ventricle and the foramen of Magendie

Correspondence to: Masahiko Wanibuchi, M.D, Department of Neurosurgery, Sapporo Medical University, Minami 1 Jo Nishi 16 Chome, Chuo-ku, Sapporo, Hokkaido 060-8543 (Japan), Tel: +81-11-611-2111, Fax: +81-11-614-1662; E-mail: wanibuti@sapmed.ac.jp

Key words: brainstem, exophytic growth, glioblastoma multiforme, medulla oblongata

Received: April 07, 2016; Accepted: May 12, 2016; Published: May 16, 2016 
Histopathological examination of paraffin-embedded tumor specimens confirmed the diagnosis of conventional GBM. A characteristic pseudopalisading appearance with geographical necrosis and pleomorphic nuclear atypia, and microvascular proliferation were observed (Figure 2A). There were no giant cells or rhabdoid components. Immunohistochemistry demonstrated substantial glial fibrillary acidic protein reactivity in the tumor cells. The proliferation rate determined by the Ki-67 index was $40 \%$ (Figure 2B). Ninety percent of the tumor cells were p53-positive. O-6-methylguanine-DNA methyltransferase (MGMT) mutation was not noted and isocitrate dehydrogenase 1 staining was negative.

Postoperatively, the patient experienced slightly worsening dysarthria and dysphagia, with transient loss of the gag reflex. Postoperative MRI showed a small residual tumor at the foramen of Magendie without dissemination (Figures 3A, 3B). Radiation therapy with a total dose of $50 \mathrm{~Gy} / 25$ fractions and concurrent treatment with temozolomide (TMZ: $75 \mathrm{mg} / \mathrm{m}^{2}$ ) were administered for 42 days. After the combined radiation and chemotherapy, MRI revealed a recurrence of the tumor in the lateral, third, and fourth ventricles (Figures $3 \mathrm{C}$, 3D). Nimusutine (ACNU: $30 \mathrm{mg} / \mathrm{m}^{2}$ ) was then added to the TMZ $\left(150 \mathrm{mg} / \mathrm{m}^{2}\right)$ chemotherapy twice. An MRI performed seven months after the operation demonstrated regression of the tumors (Figures $3 \mathrm{E}, 3 \mathrm{~F})$. After discharge from the hospital, the patient received TMZ

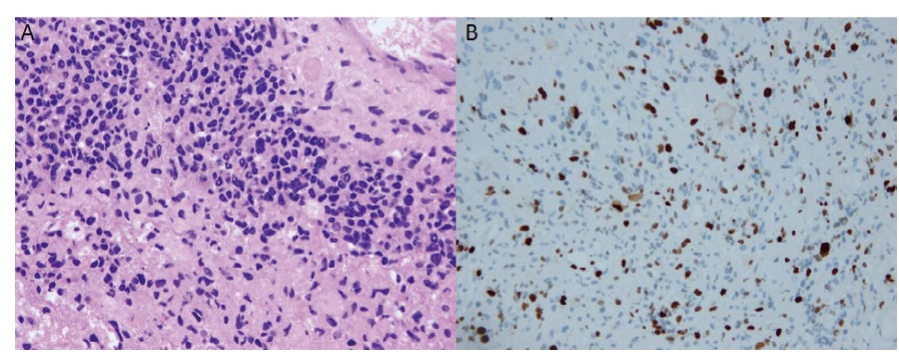

Figure 2. Micrographs of the tumor sections: A. The characteristic, pseudopalisading appearance with geographical necrosis, pleomorphic nuclear atypia, and microvascular proliferation are shown by hematoxylin and eosin staining. B. Ki-67 staining indicates a proliferation rate of $40 \%$

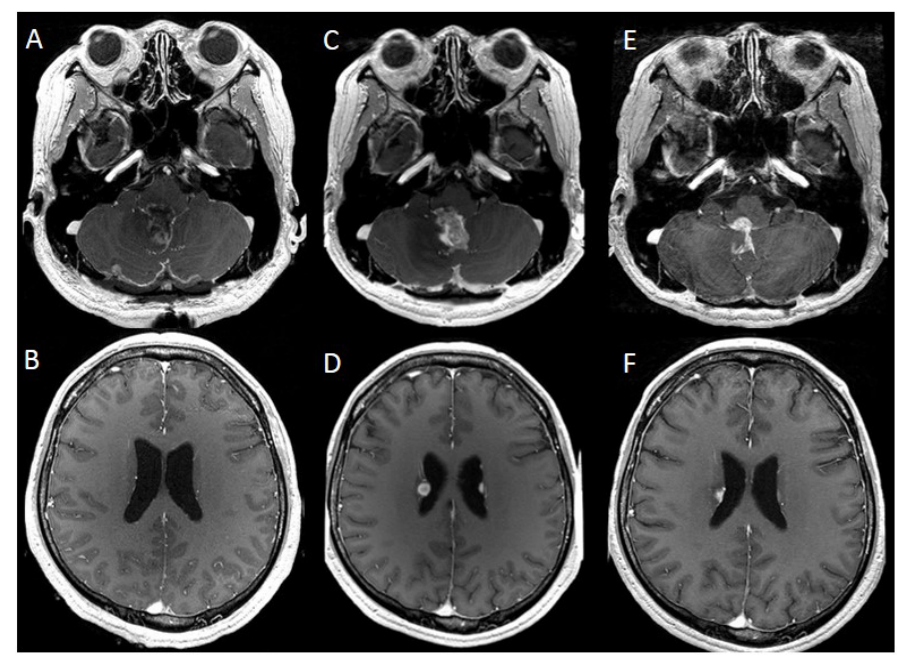

Figure 3. Postoperative magnetic resonance (MR) images: A, B. MR images enhanced with Gd-DTPA that were obtained immediately after surgery show a small residual tumor at the foramen of Magendie without dissemination. C, D. MR images taken three months post-operation reveal recurrence of the tumor in the lateral and fourth ventricles. E, F. MR images taken seven months after operation demonstrate tumor regression $\left(200 \mathrm{mg} / \mathrm{m}^{2}\right)$ treatment for five months, while local recurrence of the tumor was kept under control. He continued to independently perform his activities of daily living and his Karnofsky performance scale was maintained at $90 \%$. The patient suddenly died of respiratory arrest 12 months after his diagnosis.

\section{Discussion}

GBM seldom arises from the medulla oblongata. Brainstem gliomas constitute $10-20 \%$ of brain tumors in children [3-5] and less than $2 \%$ of brain tumors in adults [6]. The primary location of brainstem gliomas is the pons $(71.5 \%)$, followed by in the medulla oblongata (19\%), and the midbrain (7.5\%) [7]. GBMs are less common in the brainstem. Mantravadi et al. reported 12 cases in which GBMs were found in the brainstem during autopsy. They found that the primary locations were the pons (83.3\%) and the medulla oblongata (16.7\%) [2]. Only eight cases of GBMs in the medulla oblongata have been reported. Most patients with malignant brainstem gliomas died within one year and rarely survived for more than 18 months despite multimodality treatment [8]. There are no obvious reports in the literature that describe the reason that GBM seldom arises in the medulla oblongata. However, the human brain contains 100 billion neuronal cells and about ten times more glial cells [9], indicating the pons may be the most common site of GBM in the brainstem because it has a larger volume compared to the midbrain and the medulla oblongata.

A total of nine cases, including ours, have been reported of GBMs arising from the medulla oblongata in which the pathological findings were described (Table 1) [8,10-15]. Histopathological analysis indicated that all the patients had conventional GBM except for in one case reported by Luetjens et al. in which the patient had a giant cell GBM [12]. The neurological symptoms of the nine patients with GBM originating in the medulla oblongata were headache, dysphagia, hemisensory disturbance, and gait disturbance (ataxia or paresis), followed by rhinolalia, emesis, and singultus. Of the nine reported cases, exophytic growth of the tumor was observed in five cases and intrinsic growth patterns were observed in the other four cases. Surgical resections were performed in six cases (five cases of exophytic tumor growth and one case of intrinsic growth). An autopsy was carried out in all of four cases of intrinsic tumor growth.

The prognosis is much worse for patients with tumors that have intrinsic growth patterns compared to patients with tumors that have exophytic growth patterns (Figure 4). All of the patients in the intrinsic group died between 10 days and three months of diagnosis, although the patients in the exophytic group were alive for an average of 18 months. Two of the five patients in the exophytic group were alive more than two years after diagnosis. There are some similarities between the case we report here and that reported by Chotai et al. [11]. They reported a case of a patient with an exophytic GBM that originated in the medulla oblongata. Treatment with TMZ followed by radiation ( $54 \mathrm{~Gy}$ ) therapy after a subtotal resection of the tumor resulted in survival of the patient for 19 months without recurrence. The patient we describe was also treated with TMZ and radiation (50 Gy) after subtotal resection, however, postoperative MRI after three months revealed a recurrence of the tumor, so ACNU was then added to the TMZ monotherapy twice. The Ki-67 labeling index differs between our case and the case reported by Chotai et al. [11]. They reported a Ki-67 index of 10-20\%; however, we report a Ki-67 index of $40 \%$. Additionally, malignant brainstem gliomas tend to have high rates of cerebral spinal fluid seeding of tumor cells [7].

Some authors have suggested that patients with brainstem gliomas 
Table 1. Summary of GBM of medulla oblongata reported in the literature.

\begin{tabular}{|c|c|c|c|c|c|c|c|c|}
\hline Authors & Year & Age/sex & Growth & Resection & Irradiation (Gy) & ChemoTx & Ki 67 (\%) & $\begin{array}{l}\text { Outcome } \\
\text { (months) }\end{array}$ \\
\hline Queiroz et al. & 1974 & $13 / \mathrm{F}$ & Intrinsic & - & - & - & NA & $\mathrm{D}(0.3)$ \\
\hline Abbott et al. & 1991 & $15 / \mathrm{F}$ & Intrinsic & Total & - & - & NA & $\mathrm{D}(3)$ \\
\hline \multirow[t]{2}{*}{ Yoshimura et al. } & \multirow[t]{2}{*}{2003} & $28 / \mathrm{M}$ & Intrinsic & - & - & - & 20.7 & $\mathrm{D}(3.5)$ \\
\hline & & $15 / \mathrm{M}$ & Exophytic & Subtotal & 50 & $t^{* *}$ & 25.5 & $\mathrm{D}(12)$ \\
\hline Kyoshima et al. & 2004 & $55 / \mathrm{M}$ & Exophytic & Total & $++^{*}$ & TMZ & NA & $\mathrm{D}(27)$ \\
\hline Luetjens et al. & 2009 & $40 / \mathrm{M}$ & Exophytic & Total & 60 & TMZ & 15 & $\mathrm{~A}(24)$ \\
\hline Chotai et al. & 2012 & $51 / \mathrm{F}$ & Exophytic & Subtotal & 54 & TMZ & $10-20$ & A (19) \\
\hline Yoshikawa et al. & 2013 & $63 / \mathrm{F}$ & Intrinsic & - & 8 & TMZ & 23 & $\mathrm{D}(0.6)$ \\
\hline Our case & 2015 & $25 / \mathrm{M}$ & Exophytic & Subtotal & 50 & TMZ & 40 & $\mathrm{D}(12)$ \\
\hline
\end{tabular}

A: alive; D: dead; NA: not available; Tx: therapy; *: dose unclear; **: agent unclear

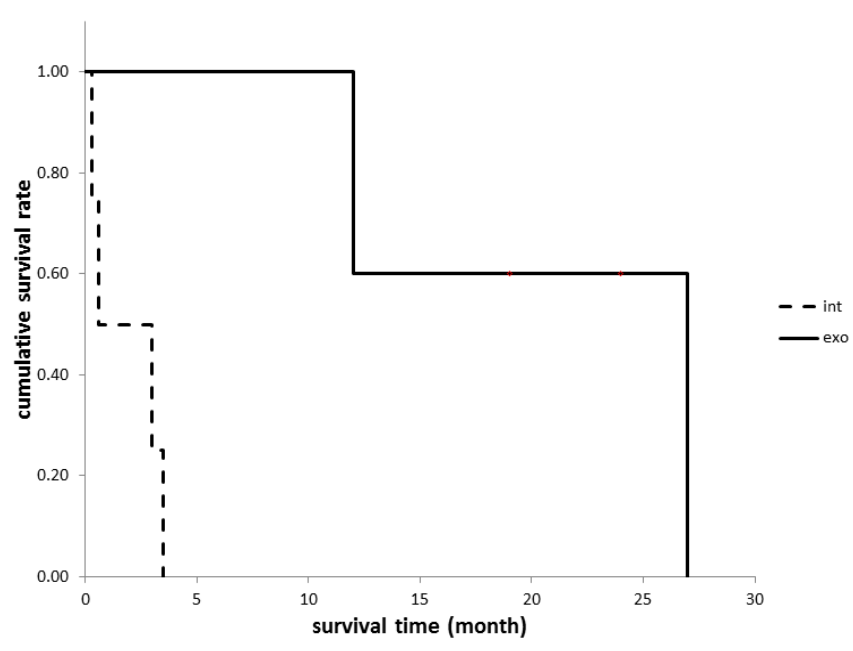

Figure 4. Overall survival according to the growth patterns of GBMs arising from the medulla oblongata. (Int: intrinsic type, Exo: exophytic type).

that exhibit dorsally exophytic growth patterns have a better prognosis than patients with tumors that have intrinsic growth patterns $[15,16]$. For example, Jallo et al. suggested that the majority of dorsally exophytic brainstem gliomas could be managed successfully with a subtotal resection [5]. Because the medulla oblongata performs vital functions $[8,10]$, the growth of intrinsic GBMs from the medulla oblongata directly damages the structures that maintain these vital functions. Therefore, the survival time of patients with this type of tumor is assumed to be shorter than that of patients with exophytic GBMs. Additionally, extensive dissection in this region often results in significant morbidity and mortality.

The brainstem has many axonal tracts that pass to the spinal cord, cerebrum, or exit as cranial nerves [3]. Epstein et al. proposed that the medulla oblongata has two anatomical barriers, the cervicomedullary and the pontomedullary junctions [17]. The former barrier includes the pyramidal decussation, the internal arcuate fibers, and the efferent fibers from the inferior olivary complex that streams from a ventromedial position toward the inferior cerebellar peduncle located posterolaterally. The latter barrier includes the transverse fibers (pontocerebellar tracts) that travel from the pontine nuclei to the contralateral middle cerebellar peduncle. Therefore, growth of the tumor originating within the medulla is limited rostrally by the pontomedullary junction and caudally by the cervicomedullary junction. Dorsally exophytic tumors in the medulla oblongata expand in the direction of least resistance, which is towards the floor of the fourth ventricle. For these reasons, dorsally exophytic brainstem gliomas have relatively good surgical outcomes because they can be successfully resected.

We have reported a rare case of dorsally exophytic GBM arising from the medulla oblongata. The patient presented in this report had a better outcome and was able to perform the activities of daily living following surgical resection and radiochemotherapy. Patients with dorsally exophytic GBMs originating in the medulla oblongata may survive for longer periods because the tumors can be more radically and safely resected than intrinsic GBMs.

\section{Conflict of interest}

The authors declare that no conflicts of interest exist.

\section{References}

1. Walter J, Koch A, Herbold C, Schiffler S, Reichart R, et al (2013) Multifocal Glioblastoma Multiforme in the Posterior Fossa Mimicking Cerebral Metastases: Case Presentation and Review of the Current Literature. J Neurol Surg A Cent Eur Neurosurg 74 Suppl 1: e30-35. [Crossref]

2. Mantravadi RV, Phatak R, Bellur S, Liebner EJ, Haas R (1982) Brain stem gliomas: an autopsy study of 25 cases. Cancer 49: 1294-1296. [Crossref]

3. Donaldson SS, Laningham F, Fisher PG (2006) Advances toward an understanding of brainstem gliomas. J Clin Oncol 24: 1266-1272. [Crossref]

4. Ueoka DI, Nogueira J, Campos JC, Maranhão Filho P, Ferman S, et al. (2009) Brainstem gliomas--retrospective analysis of 86 patients. J Neurol Sci 281: 20-23. [Crossref]

5. Jallo GI, Biser-Rohrbaugh A, Freed D (2004) Brainstem gliomas. Childs Nerv Syst 20: 143-153. [Crossref]

6. White HH (1963) Brain stem tumors occurring in adults. Neurology 13: 292-300 [Crossref]

7. Nishio S, Fukui M, Tateishi J (1988) Brain stem gliomas: a clinicopathological analysis of 23 histologically proven cases. J Neurooncol 6: 245-250. [Crossref]

8. Kyoshima K, Sakai K, Goto T, Tanabe A, Sato A, et al. (2004) Gross total surgica removal of malignant glioma from the medulla oblongata: report of two adult cases with reference to surgical anatomy. J Clin Neurosci 11: 75-80. [Crossref]

9. Noctor SC, Martínez-Cerdeño V, Kriegstein AR (2007) Contribution of intermediate progenitor cells to cortical histogenesis. Arch Neurol 64: 639-642. [Crossref]

10. Abbott R, Shiminski-Maher T, Wisoff JH, Epstein FJ (1991) Intrinsic tumors of the medulla: surgical complications. Pediatr Neurosurg 17: 239-244. [Crossref]

11. Chotai SP, Moon HJ, Kim JH, Kim JH, Kwon TH (2012) Primary glioblastoma multiforme of medulla oblongata: Case report and review of literature. Asian $J$ Neurosurg 7: 36-38. [Crossref]

12. Luetjens G, Mirzayan MJ, Brandis A, Krauss JK (2009) Exophytic giant cell glioblastoma of the medulla oblongata. J Neurosurg 110: 589-593. [Crossref] 
13. Queiroz Lde S, da Cruz Neto JN, Lopes de Faria J (1974) Glioblastoma multiforme of the medulla oblangata. Acta Neuropathol 29: 355-360. [Crossref]

14. Yoshimura J, Onda K, Tanaka R, Takahashi H (2003) Clinicopathological study of diffuse type brainstem gliomas: analysis of 40 autopsy cases. Neurol Med Chir (Tokyo) 43: 375-382. [Crossref]

15. Yoshikawa A, Nakada M, Watanabe T, Hayashi Y, Sabit H, et al. (2013) Progressive adult primary glioblastoma in the medulla oblongata with an unmethylated MGMT promoter and without an IDH mutation. Brain Tumor Pathol 30: 175-179. [Crossref]

16. Cohen ME, Duffner PK, Heffner RR, Lacey DJ, Brecher M (1986) Prognostic factors in brainstem gliomas. Neurology 36: 602-605. [Crossref]

17. Epstein FJ, Farmer JP (1993) Brain-stem glioma growth patterns. J Neurosurg 78: 408412. [Crossref]

Copyright: $\mathbb{0} 2016$ Horita Y. This is an open-access article distributed under the terms of the Creative Commons Attribution License, which permits unrestricted use, distribution, and reproduction in any medium, provided the original author and source are credited. 